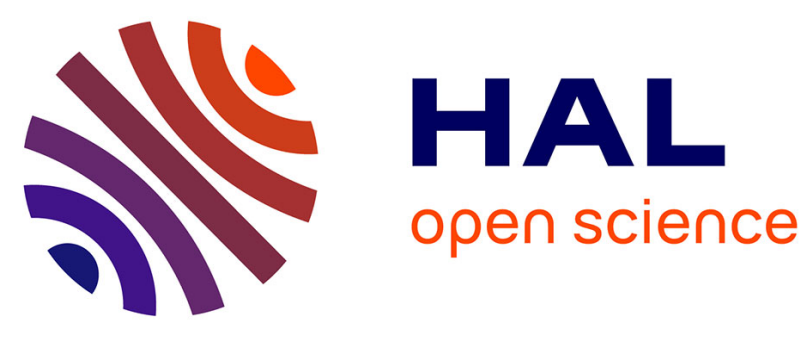

\title{
Stretchable Strain Sensors for Human Movement Monitoring
}

Abhishek Singh Dahiya, Thierry Gil, Nadine Azemard, Jérôme Thireau, Alain Lacampagne, Aida Todri-Sanial, Benoît Charlot

\section{- To cite this version:}

Abhishek Singh Dahiya, Thierry Gil, Nadine Azemard, Jérôme Thireau, Alain Lacampagne, et al.. Stretchable Strain Sensors for Human Movement Monitoring. DTIP 2020 - 22nd Symposium on Design, Test, Integration \& Packaging of MEMS and MOEMS, Jun 2020, (Virtual ), France. 10.1109/DTIP51112.2020.9139154 . hal-02903236

\section{HAL Id: hal-02903236 \\ https://hal.science/hal-02903236}

Submitted on 21 Jul 2020

HAL is a multi-disciplinary open access archive for the deposit and dissemination of scientific research documents, whether they are published or not. The documents may come from teaching and research institutions in France or abroad, or from public or private research centers.
L'archive ouverte pluridisciplinaire HAL, est destinée au dépôt et à la diffusion de documents scientifiques de niveau recherche, publiés ou non, émanant des établissements d'enseignement et de recherche français ou étrangers, des laboratoires publics ou privés. 


\section{Stretchable Strain Sensors for Human Movement Monitoring}

\author{
Abhishek Singh Dahiya \\ BEST group, James Watt \\ School of Engineering \\ University of Glasgow \\ Glasgow, UK \\ abhisheksingh.dahiya@gla \\ sgow.ac.uk \\ Alain Lacampagne \\ PhyMedExp \\ Université de Montpellier, \\ CNRS, INSERM \\ Montpellier, France \\ alain.lacampagne@inserm. \\ fr
}

\author{
Thierry Gil \\ LIRMM \\ Université de Montpellier, \\ CNRS \\ Montpellier, France \\ Thierry.gil@lirmm.fr
}

Aida Todri-Sanial
LIRMM
Université de Montpellier,
CNRS
Montpellier, France
aida.todri@lirmm.fr

\author{
Nadine Azemard \\ LIRMM \\ Université de Montpellier, \\ CNRS \\ Montpellier, France \\ nadine.azemard@lirmm.fr \\ Benoit Charlot \\ IES \\ Université de Montpellier, \\ CNRS \\ Montpellier, France \\ benoit.charlot@umontpelli \\ er.fr
}

\author{
Jerome Thireau \\ PhyMedExp \\ Université de Montpellier \\ CNRS, INSERM \\ Montpellier, France \\ jerome.thireau@inserm.fr
}

\begin{abstract}
Stretchable strain sensors based on organic/inorganic hybrid NanoComposite (NC) have gained wide interest owing to their potential application in health diagnosis, soft robotics, and wearable electronics. This paper describes a facile strategy of micromolding-in-capillary process to fabricate stretchable strain sensors wherein, the sensing material (i.e. one-dimensional (1D) material) is wrapped within silicone rubber (Dragon SkinTM (DS)) to form a sandwich-like structure. The fabricated strain sensors exhibit superb stretchability (wide strain sensing range of up to $180 \%$ ), and moderately high sensing performance with outstanding stability and durability. They can be applied for human movement monitoring such as finger movements to enable human physiological parameters to be registered and analyzed continuously.
\end{abstract}

Keywords - Stretchable strain sensors, human movement detection, silicone rubber, carbon nanotubes, silver nanowires

\section{INTRODUCTION}

Piezoresistive-based stretchable strain sensors are gaining a lot of interest as they provide high sensitivity with simple device design and readout circuits [1]. They have gained significant interest recently because of the wide range of wearable applications they enable which includes heathmonitoring [2], human movement and motion detection, and intelligent robots [1]. When a wearable sensor is attached to human skin or robots, they need to be well adhered and conformal to moving surfaces. Further, they should stretch along with the moving surfaces such as human skin to provide comfort to the user. Looking into these aspects, some of the important requirements for a wearable strain sensor are: (i) high stretchability, (ii) high sensitivity, (iii) biocompatibility, and (iv) insensitivity to biological fluid such as sweat.

To address these needs, organic/inorganic hybrid 'nanocomposite (NC) materials' are being investigated [3][5]. This new class of stretchable material is a mixture of viscoelastic polymer matrix (organic) and conductive fillers (inorganic). These NC materials provide reliable sensing signals while enabling movement and comfort for users. This class of material is unique as it enjoys many desirable features of organic materials (flexibility, biocompatibility, processability) yet, it adds electrical conductivity and piezoresistive property of the conductive fillers (generally nanomaterials) to design stretchable sensors with skin-line conformability and stretchability [6]. Many methods have been reported to form percolated conductive networks throughout the polymer matrix. However, issues such as the large amount of filler requirement, hysteresis, poor sensitivity etc. still exist. Research efforts are on-going to develop novel fabrication approaches and NC materials to resolve these issues.

Innovative device fabrication approaches have been experimented to develop highly sensitive stretchable strain sensors such as pre-stretching [7], 3D printing [8], direct ink writing [9] and microfluidic techniques [10]. For instance, exploiting 3D printing method [8] fabricate sensitive strain sensors with conductive carbon grease materials to obtain a high stretchability of $400 \%$. More recently, microfluidic based approach was used to fabricate high performance strain sensor. In this fabrication approach, networks of 1D materials, such as carbon nanotubes (CNTs) or silver (Ag) nanowires (NWs), on top of a stretchable substrate are realized in a micro/macro channels, which offers an alternative way to realize stretchable strain sensors with high gauge factor (GF) [1], [10]. Using this fabrication strategy, Han et al. [1], created a NW-based strain sensor by hybridizing brittle metal nanowires (Ag NWs, Copper NWs, or CNTs) and a conductive organic solution with poly $(3,4-$ ethylenedioxythiophene):polystyrene sulfonate (PEDOT:PSS). Nanowire microfluidic hybrid strain sensors exhibit record-high values for both GF and stretchability. This is because the advantages of both materials have been coupled. The brittleness of the NWs endows the strain sensor with an outstanding sensitivity under a small stretchable load, and the conductive organic solution significantly enhances the deformation endurance of the device.

In this work, we present a new, facile, and cost-effective strategy to construct organic/inorganic hybrid NC based stretchable strain sensors. We have developed a 


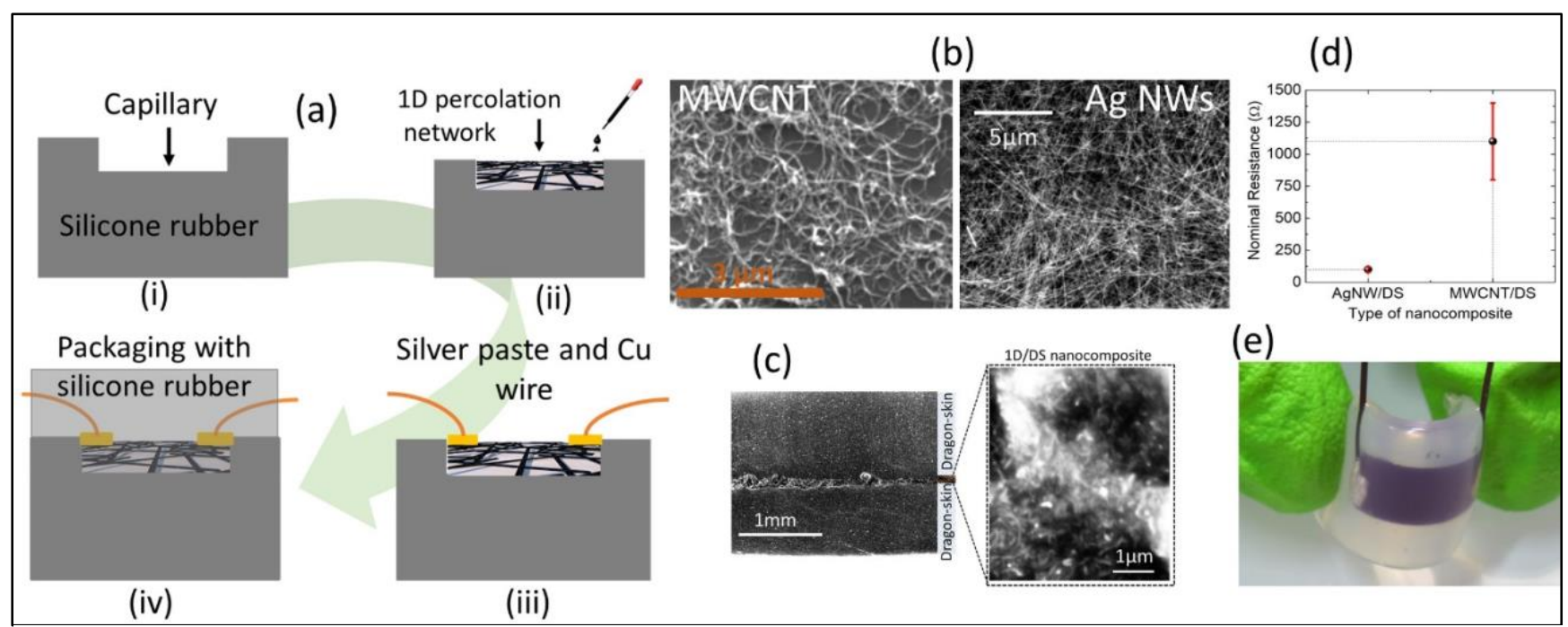

Figure 1. (a) Schematic of the micromolding-in-capillary (MIMIC) fabrication process of the stretchable strain sensors, (b) SEM images of the 1D materials (MWCNTs and Ag NWs), (c) cross-sectional SEM images of the sensor, (d) average nominal device resistance of the 1D material / DS nanocomposite materials, and (e) optical micrograph of a MWCNT/DS sensor.

micromolding-in-capillary (MIMIC) process in which the sensing material i.e. one-dimensional (1D) nanomaterial is wrapped within dragon-skin (DS) polymer to form a sandwich-like structure (Fig.1 (a)). The unique advantages of the developed fabrication process are the use of significantly less amount of filler material to obtain high NC conductivity as compared to conventional NC materials. Secondly, we obtain excellent dispersal of filler material in polymer matrix without any chemical or physical treatments.

\section{EXPERIMENTAL SECTION}

\section{A. One-dimensional materials}

Multi-walled carbon nanotubes (MWCNTs) are purchased from cheaptubes.com (purity of $>95 \mathrm{wt} \%$ ) with 8$15 \mathrm{~nm}$ outer diameter and length of $10-50 \mu \mathrm{m}$. Silver nanowires (Ag NWs) are purchased from Sigma Aldrich. The diameter and length of the NWs are $35 \pm 5 \mathrm{~nm}$ and $25 \pm 5 \mu \mathrm{m}$, respectively. Fig.1 (b) shows the scanning electron microscopy (SEM) images of the materials. Dragon Skin ${ }^{\mathrm{TM}}$ silicones are used for a variety of applications, ranging from creating skin effects and other movie special effects to making production moulds for casting a variety of materials. Due to their superior physical properties and flexibility, they are also used for medical prosthetics and cushioning applications. It is purchased from Smooth-On company.

\section{B. Synthesis of $1 D$ material solution and Dragon Skin ${ }^{\mathrm{TM}}$ curing process}

MWCNTs/IPA solution: Mix CNTs in isopropanol (IPA) according to the desired weight ratio. The optimized weight percent is $0.5 \mathrm{wt} \%$. Place the solution in ultrasonicator and let it for one hour to detangle the nanotubes to form uniform suspension. Ag NWs were used as received $(5 \mathrm{mg} / \mathrm{ml}$ in ethanol).

Dragon Skin ${ }^{\mathrm{TM}}$ : As a stretchable organic part, DS elastomer is made using part A and part B. The mass ratio between the two is $1: 1$. Then, this mixture is thoroughly mixed and degassed for $2 \mathrm{~min}$ in a desiccator. Following this step, an $\approx 1000 \mu \mathrm{m}$ thick DS layer is drop casted over Kapton tape or SU-8 rigid pattern. The DS layer is kept at room temperature for $45 \mathrm{~min}$ to finish the curing process.

\section{Functional characterization of the sensors}

The electromechanical properties of the developed strain sensors are subsequently characterized using our custombuilt test-bench set up. The change in resistance while stretching the DS substrate using a linear stage is monitored using LabVIEW controlled Keithley 2400 source meter. The linear stage is controlled using the E-861 PiezoWalk ${ }^{\circledR}$ NEXACT ${ }^{\circledR}$ Controller. The stretch rate was varied between $0.1 \mathrm{~mm} / \mathrm{s}$ to $10 \mathrm{~mm} / \mathrm{s}$.

\section{RESULTS AND DISCUSSIONS}

The fabrication method of strain sensors is inspired from a micromolding-in-capillary (MIMIC) [11] process, schematically shown in the Fig.1 (a). In the first step, grooved bottom DS plate is realized using a standard moulding process (see experimental section for curing process of DS). The groove dimensions and depth can be tuned according with the size and thickness of polyimide or SU-8 (epoxybased negative photoresist) layer. Following, a drop of the 1D material suspension (approximately $100 \mu \mathrm{L}$ ) is introduced at the DS mould tip. Two different 1D sensing materials have been exploited to fabricate and study strain sensing performance of such device structure, namely MWCNTs and Ag NWs. The suspension filled the channels of the DS mould through capillary forces. In the MIMIC process, the rate of capillary filling depends on the kinematic viscosity, surface tension of the liquid, and section size of the capillary [11], [12]. In the third step, copper $(\mathrm{Cu})$ wires are attached using silver epoxy paste. In the last step, "sandwich" structure is realized by pouring another layer of DS. The top DS layer will fully and firmly package the 1D sensing material, thus only sensing material move with the DS deformation

The cross-sectional SEM images of the packaged 1D sensing material, between two DS layers, is shown in the Fig.1 (c). It can be seen using SEM images that the 1D sensing material is uniformly dispersed and firmly packaged within DS polymer. Fig.1 (d) presents the bar graph showing 
(a)

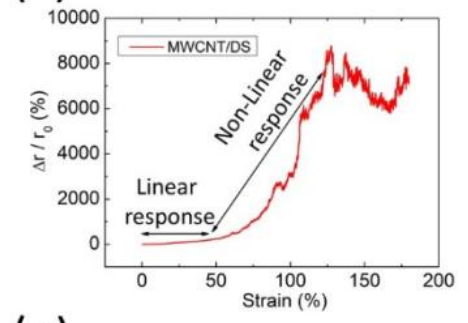

(e)

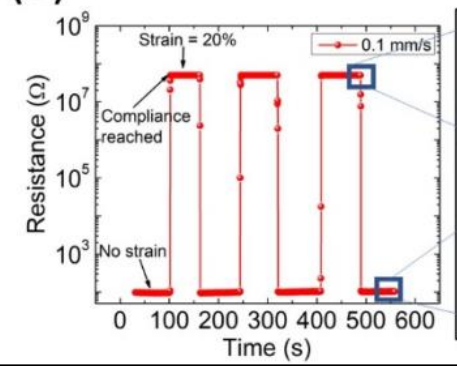

(b)
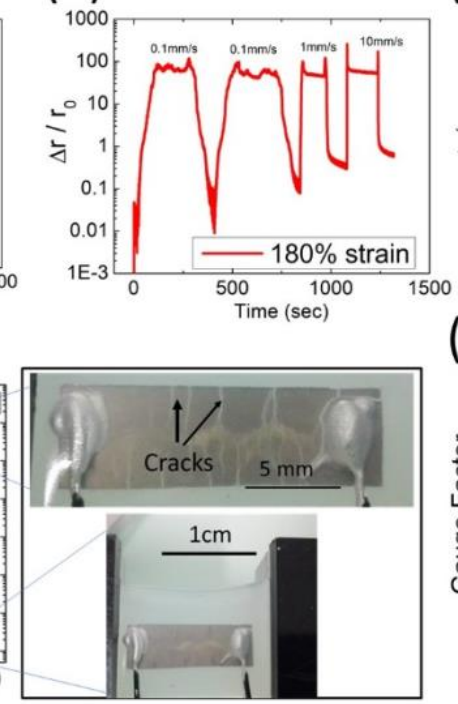

(c)

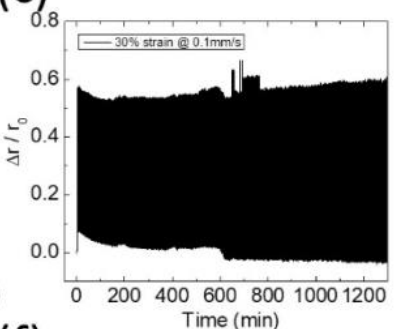

(f)

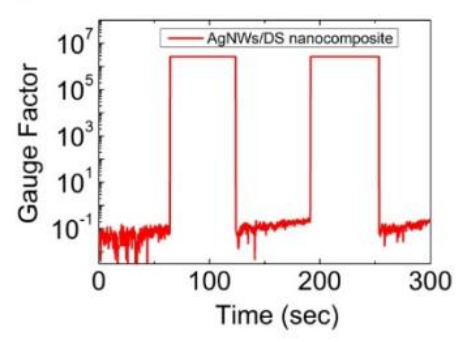

(d)

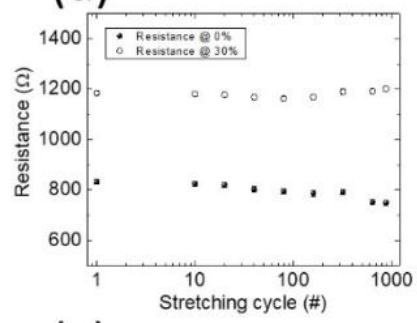

(g)

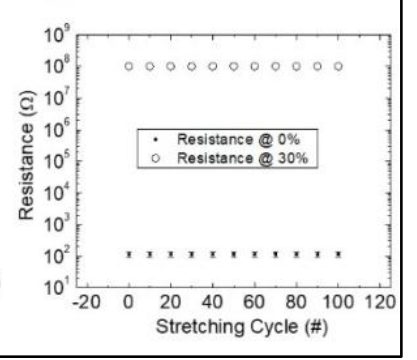

Figure 2. Electromechanical characterization results of the nanocomposite-based sensor: (a-d) MWCNT/DS nanocomposite and (e-g) AgNWs/DS nanocomposite.

average nominal resistance values obtained using MWCNTs and Ag NWs sensing materials. As expected, Ag NWs/DS NC material exhibit lower resistance values $(\sim 100 \Omega)$ compared to MWCNT/DS NC $\left(\begin{array}{lll}\sim 1 & \mathrm{k} \Omega\end{array}\right)$. The electromechanical properties of the developed strain sensors subsequently characterized in detail using our custom-built test bench. The change in resistance while uniaxially stretching the DS substrate using a linear stage is monitored using LabVIEW controlled keithley 2400 source meter. The sensitivity of sensor is quantified by calculating a figure of merit, i.e. the Gauge Factor (GF), which is defined as GF = $\left(\Delta \mathrm{r} / \mathrm{r}_{0}\right) /\left(\Delta \mathrm{l} / \mathrm{l}_{0}\right)$, where $\mathrm{r} 0$ and $\Delta \mathrm{r}\left(\mathrm{r}-\mathrm{r}_{0}\right)$ correspond to the original resistance and the change in resistance under stretching, respectively and $\Delta \mathrm{l}$ is the change in the length and 10 is the initial length. Fig. 2 (a-d) shows the electromechanical characterization results for MWCNT/DS composite. As shown in the Fig.2 (a), the MWCNT/DS sensor shows that at small strain levels of up to $45 \%$ (i.e. 4.5 $\mathrm{mm}$ of stretching), the device showed almost linear change of resistance with strain applied. Beyond $45 \%$ strain, the resistance increases rapidly up to $120 \%$ strain. The corresponding GF at $120 \%$ strain is 60 . It is to note that the device showed no further increase in resistance with further stretching up to $180 \%$. The effect of strain rate and stability test results are shown in Fig.2 (b) and 2 (c-d), respectively. The strain rate is varied from $0.1 \mathrm{~mm} / \mathrm{s}$ to $10 \mathrm{~mm} / \mathrm{s}$ with a step increase of one order. Fig.2 (b) presents $\Delta \mathrm{r} / \mathrm{r}$ o values when the strain sensor was stretched with different strain rates keeping maximum strain constant $(180 \%)$. Devices showed good recoverability at lower strain rate. At higher stain rates, small hysteresis and shoulder peaks has appeared. Similar shoulder peaks has been observed in other CNT/polymer composites [4], which is mainly attributed to the competition between the destruction and reconstruction of conductive networks during cyclic loadings. However, further investigations are needed to confirm the proposed mechanism. The stability of the MWCNT/DS NC-based strain sensors were studied by loading and unloading stretching cycles for 30\% strain (900 stretch cycles at a strain rate of $0.1 \mathrm{~mm} / \mathrm{s}$ ). The results are shown in Fig.2 (c-d). It is observed that the maximum value of $\Delta \mathrm{r} / \mathrm{r}$ r remains unchanged with stretch cycles. These results show that MWCNT/DS composite possess superior stability and repeatability, which could be used as smart strain sensors to continuously monitor human movements. Even after $24 \mathrm{~h}$ of continuous operation, the device nominal resistance remains same which shows the high durability of the fabricated devices. The electromechanical characterisation results for AgNWs/DS composite are shown in Fig.2 (e-g). A very high GF of $>106$ is achieved using AgNWs/DS based sensor at $30 \%$ strain with good stability (data for 100 stretch cycles are shown). The occurrence and propagation of the cracks in the concentrated strained areas of the brittle conductive layers when the composites are stretched, is the proposed working mechanism for showing high sensitivity when using Ag nanomaterial as filler material [13]. The appearance and propagation of the cracks lead to a remarkable increase in electrical resistance (see optical images in Fig.2 (e)).

As MWCNT/DS composite shows a large working window (stretchable up to $180 \%$ ), it can be a promising platform for application as sensitive stretchable strain sensors for human movement monitoring. Subsequently, as a proofof-concept demonstration, we used the MWCNT/DS sensor to monitor the bending movement of the human index finger. To monitor the finger movements, the MWCNT/DS device is mounted on a nitrile glove that is subsequently worn by a volunteer (Fig.3 (a)). Unambiguous stepwise resistance changes are recorded while the finger is bent gradually (Fig.3 (a)). Finally, the strain sensor is mounted on a nitrile glove (on index finger) and connected with a single-board microcontroller (Arduino UNO) and light emitting diodes (LEDs), as shown in the Fig.3 (b). The microcontroller is programmed to light LEDs when strain sensor resistance increases. When the finger is bended and sensor is stretched, its resistance increases. The voltage at the terminals of the resistive sensor is measured through an analog-to-digital converter. The greater the resistance, the greater the voltage 


\section{(a)}

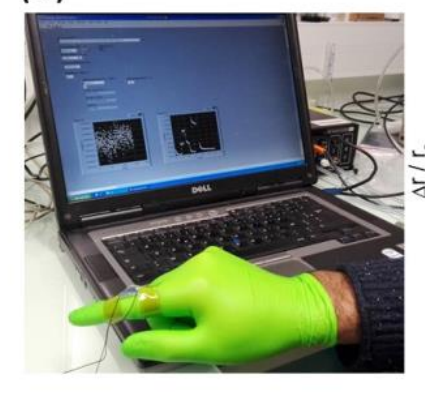

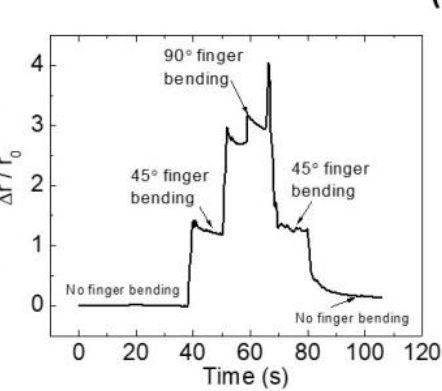

(b)

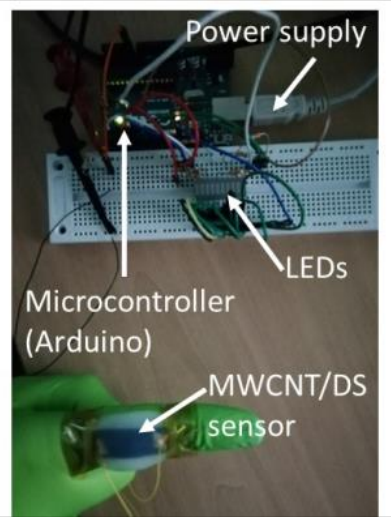

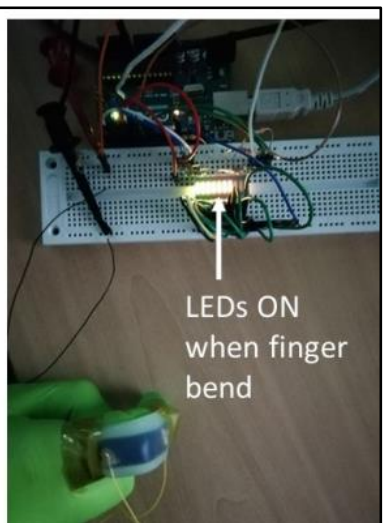

Figure 3. Finger movement monitoring: (a) optical image of the MWCNT/DS sensor on index finger and $\Delta \mathrm{r} / \mathrm{r}$ o variation with finger bending. (b) Illustration of finger movement monitoring with an integrated electronic system constructed using a microcontroller, LEDs and strain sensor placed on index finger.

measured at the terminals and the greater the number of illuminated LEDs.

\section{CONCLUSIONS}

In summary, we proposed a simple and cost-effective fabrication process to produce sensitive and stretchable strain sensors for human movement sensing. The strain sensors include macro channels filled with 1D inorganic materials (MWCNTs or AgNWs) and organic silicone polymer. The organic/inorganic hybrid sandwich structure provides percolation paths for electrical conduction at the cost of a significantly low amount of filler material. The MWCNT/DS based sensors were tested to demonstrate real-world applications such as monitoring human finger movements. Using a single-board microcontroller, we have demonstrated changes in LEDs colours with the applied strain (no colour at zero strain, yellow at a lower level of strain and red colour at higher values of strain). This organic/inorganic hybrid strain sensor may advance the realization of future soft electronic devices, such as smart robotics, electronic skin, human motion, health-monitoring systems, and human-machine interactive systems.

\section{ACKNOWLEDGMENT}

This work was financially supported through EU H2020 SmartVista project, which has received funding from the European Union's Horizon 2020 research and innovation programme under the grant agreement No. 825114.

\section{REFERENCES}

S. Han et al., "Multiscale nanowire-microfluidic hybrid strain sensors with high sensitivity and stretchability," npj Flex. Electron., vol. 2, no. 1, p. 16, 2018, doi: 10.1038/s41528-0180029-x.

[2] A. S. Dahiya et al., "Energy Autonomous Wearable Sensors for Smart Healthcare: A Review," J. Electrochem. Soc., vol. 167, no. 3, p. 37516, Jan. 2020, doi: 10.1149/2.0162003jes.

[3] R. Zhang et al., "Strain sensing behaviour of elastomeric composite films containing carbon nanotubes under cyclic loading," Compos. Sci. Technol., vol. 74, pp. 1-5, 2013, doi: 10.1016/j.compscitech.2012.09.016.

[4] H. Yang, X. Yao, L. Yuan, L. Gong, and Y. Liu, "Strain-sensitive electrical conductivity of carbon nanotube-graphene-filled rubber composites under cyclic loading," Nanoscale, vol. 11, no. 2, pp. 578-586, 2019, doi: 10.1039/c8nr07737a.

[5] J. Chen et al., "Strain sensing behaviors of stretchable conductive polymer composites loaded with different dimensional conductive fillers," Compos. Sci. Technol., vol. 168, no. October, pp. 388396, 2018, doi: 10.1016/j.compscitech.2018.10.025. wearable wireless biosensors," Biosens. Bioelectron., vol. 141, no. June, p. 111422, 2019, doi: 10.1016/j.bios.2019.111422.

[7] Kyun Kyu Kim et al., "Highly Sensitive and Stretchable Multidimensional Strain Sensor with Prestrained Anisotropic Metal Nanowire Percolation Networks," Nano Lett., vol. 15, p. 5240, 2015, doi: 10.1021/acs.nanolett.5b01505.

[8] J. T. Muth, D. M. Vogt, R. L. Truby, D. B. Kolesky, R. J. Wood, and J. A. Lewis, "Embedded 3D Printing of Strain Sensors within Highly Stretchable Elastomers," Adv. Mater., vol. 26, pp. 63076312, 2014, doi: 10.1002/adma.201400334.

[9] J. W. Boley, E. L. White, G. T. C. Chiu, and R. K. Kramer, "Direct writing of gallium-indium alloy for stretchable electronics," $A d v$. Funct. Mater, vol. 24, no. 23, pp. 3501-3507, 2014, doi: 10.1002/adfm. 201303220.

[10] Z. Pei et al., "Highly Sensitive, Stretchable Strain Sensor Based on Ag@COOH-Functionalized CNTs for Stroke and Pronunciation Recognition," Adv. Electron. Mater., vol. 1900227, pp. 1-10, 2019, doi: 10.1002/aelm.201900227.

[11] E. Kim, Y. Xia, and G. M. Whitesides, "Micromolding in capillaries: Applications in materials science," J. Am. Chem. Soc., vol. 118, no. 24, pp. 5722-5731, 1996, doi: 10.1021/ja960151v.

[12] B. Ha and S. Jo, "Hybrid Ag nanowire transparent conductive electrodes with randomly oriented and grid-patterned $\mathrm{Ag}$ nanowire networks," Sci. Rep., vol. 7, no. 1, pp. 1-8, 2017, doi: 10.1038/s41598-017-11964-w.

[13] J. Chen et al., "An overview of stretchable strain sensors from conductive polymer nanocomposites," J. Mater. Chem. C, vol. 7, no. 38, pp. 11710-11730, 2019, doi: 10.1039/c9tc03655e. 